International Journal of Engineering \& Technology, $7(2.25)(2018)$ 10-13
International Journal of Engineering \& Technology
Website: $w$ ww.sciencepubco.com/index.php/IJET
Research paper

\title{
A study on wavelet analysis of SSVEP Signals
}

\author{
Bincy Babu*, R Chandrasekaran, Josline Elsa Joseph, Thella Shalem Rahul, T R Thamizhvani, \\ A Josephin Arockia Dhivya \\ Department of Biomedical Engineering, Vels Institute of Science, Technology and Advanced Studies (VISTAS), \\ Pallavaram, Chennai-600117, India \\ *Corresponding author E-mail: bincyrijo.se@velsuniv.ac.in
}

\begin{abstract}
Almost every Brain Control Interfcae (BCI) system is framed based on Steady State Visual Evoked Potential (SSVEP) which is predicted through distinguishing overriding frequency components in Electroencephalography (EEG) signals. The proposed system aims in accurate feature extraction of SSVEP signals. Power spectral analysis and wavelet analysis are done for feature analysis. The feature set variation for male and female subjects are obtained. Compared power spectral estimation and wavelet analysis, merits and demerits of each approach can be identified from the outcomes. It offers a theoretical reference of practical choice for BCI application.
\end{abstract}

Keywords: BCI, EEG; Power spectral density; SSVEP; Wavelet

\section{Introduction}

The Electroencephalography (EEG) is magnitude and direction of electrical activity of brain, obtained by placing electrodes along scalp. It processes the voltage variations causing from ionic current present within neurons of brain [1]. Diagnostic applications of ECG emphasis either on spectral content of EEG or event related potentials. Steady State Visual Evoked Potential (SSVEP) is generally used with EEG investigation related to visual response. These are signals that obtained from brain due to normal responses to visual stimulation [2]. The brain produces electrical activity when visual stimulus at particular frequency rage is allowed to excite retina. The frequency range of stimulus is $3.5 \mathrm{~Hz}$ to $75 \mathrm{~Hz}$ and the brain makes electrical response to same or multiple of frequency of stimulus.

SSVEP have an important role in EEG analysis and study because of comparative resistance to artifacts and good Signal to Noise Ratio (SNR). SSVEP usually arise in parietal and occipital lobes as a result of repetitive series of visual stimulus. The development of SSVEP is depending on evoked intensity and frequency. It permits to gather a bulky number of trials within a short interval. SSVEP plays a vital role in Brain Control Interface (BCI), which is an emergent research area contributes to neurorehabilitation [3]. A typical BCI system comprises of different stages such as acquisition of data, preprocessing, finally extraction and classification of features. A standard BCI system usually includes multiple stages, including data acquisition, data preprocessing, feature extraction, feature classification, and command translation. Feature extraction is an important role in signal analysis and study. Different methods are applied in classification of signals. Fast Fourier Transform (FFT) method requires extensive data segments and it is also a fast computation technique. For real time applications, if frequency resolution of a data segment is limited then available stimulation frequencies are always limited [5-8]. A modifiable window type Fourier analysis is wavelet transform. This approach has better frequency time resolution compared with Fourier transform and finds in applications for non-stationary signals but not for nonlinear. An extended approach is wavelet decomposition, which decomposes raw signal into different wavelet packets [9, 10]. For real applications, Continuous Wavelet Transform (CWT) is effective compared to other approaches [11].

Ensemble Empirical Mode Decomposition (EEMD) was established to avoid mode mixing issue of Empirical mode decomposition. Compared to wavelet transform, EEMD is appropriate for non-stationary signals but have demerit that it needs more computation time [12]. Hilbert transform calculate phases of SSVEP have an advantage that it requires shorter data length [13, 14]. The combination of Hilbert transform and Empirical Mode Decomposition is Hilbert-Huang Transform (HHT). It is applicable for not only nonlinear but also non-stationary signal processing. As same as that of Empirical Mode Decomposition, it has higher computational time [15]. In combined approach of Huang Transform and Maximum contrast combination, the input data is filtered by maximum contrast combination, power was calculated at stimulation frequency and computed phases by Huang transform [16]. Minimum energy combination method filters raw SSVEP signals. Then power is computed at each stimulation [17]. In study of SSVEP feature extraction, spectral F-test is adapted which is built by power spectral analysis $[18,19]$. Matched filter detector is applied to find power by calculating inner product of normalized complex signal and SSVEP. The computation model of this approach is same as that of Canonical correlation analysis. It have an advantage that it needs only less computational time compared to other techniques but adaptable for only single channel analysis [20].

\section{Methodology}

This work proposes different SSVEP signal compression techniques. Fig.1 shows block diagram of proposed analysis system. It 
comprise of two sections: Power Spectral Density (PSD) and wavelet transform. FFT, Burg covariance and Yule walker are applied for power spectral density analysis. Haar and Daubechies are taken for wavelet transform.

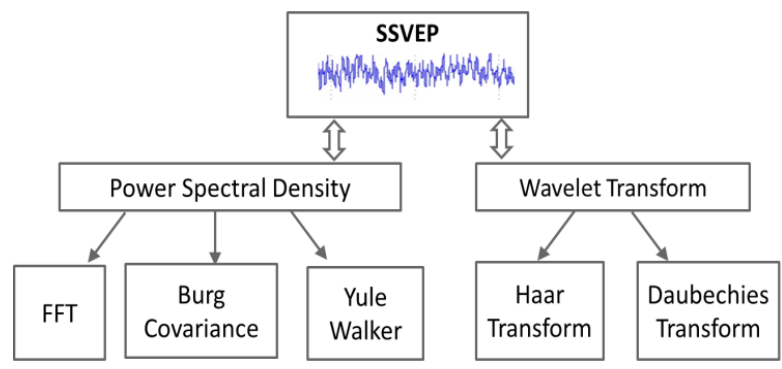

Fig. 1 Proposed system .

\subsection{Power Spectral Density}

PSD illustrates energy variations as frequency function. It provides details of frequency variations such as which is strong or weak. PSD is independent of time for some forms of random data; so it is deterministic. FFT algorithm results input data into different frequency components by sampling signal over a time period. Thus obtained components have their own phase and amplitude at distinctive frequencies. Burg Covariance method approximates PSD of input signal. Burg algorithm influences Levinson recursion in which it minimizes backward and forward prediction of least square errors, results in harmonic mean. It obtains better resolution than Yule Walker method especially for short data. A special instance of least square algorithm is covariance technique, which has improved performance to Burg algorithm. Burg Covariance method not only minimizes all prediction errors but also free from splitting of spectral lines. One of the main advantages is that it gives less distortion. Yule Walker method also called autocorrelation technique that reduces forward prediction error. Compared to covariance, It results in weakened performance. Yule Walker algorithm can be competently solved by using Levinson recursion. It performs effectively for large data but not for short data.

\subsection{Wavelet Transform}

It is a signal processing tool in which signal is decomposes into details as time function. It is helpful in characterization of very short events. The main advantage of wavelet transform is localized basis and faster computational speed. Localized basis are ideal for analysis of real time physical situations. A rescaled sequence of square shaped functions called Haar wavelet. Both Fourier analysis and haar transform permits target function over a particular interval. It is simplest wavelet but not continuous and there by not differentiable so supportive in analysis of sudden transition of signals. It is an orthogonal wavelet transform, which is characterized by a maximal number of vanishing moments for some given support. With each wavelet type of this class, there is a scaling function (called the father wavelet) which generates an orthogonal multi resolution analysis. The Daubechies wavelet transforms are defined in the same way as the Haar wavelet transform by computing running averages and differences via scalar products with scaling signals and wavelets. The main difference between both is in the way of defining wavelets and scaling signals. In case of Daubechies transforms, the wavelets and scaling signals have marginally longer support compared to haar. It yields differences and average values by taking signal values. This slender change can results a tremendous enhancements in the performance of these transforms. They offer an effective tool in execution of signal processing tasks. It comprises noise removal and compression for images and audio signals.

\section{Result and discussion}

The SSVEP data signals are taken from the database-physionet [21], which consists of signals form 11 subjects including $3 \mathrm{fe}-$ males and 8 males. Subjects who have age between 24 and 39 were exposed to non-overlapping flickering lights. The frequencies chosen are $6.66 \mathrm{~Hz}, 7.5 \mathrm{~Hz}, 8.57 \mathrm{~Hz}, 10 \mathrm{~Hz}$ and, $12 \mathrm{~Hz}$. For an adaptation period, subject is exposed to 8 flickering $(5 \mathrm{sec})$ where the adaptation frequency varying 6.66 to $12 \mathrm{~Hz}$. Fig. 2 and Fig. 3 shows two different original signals. The PSD is estimated for SSVEP signals, Fig. 4 shows FFT power spectral density estimation. Fig.5 shows Burg Covariance and Fig.6 Yule Walker PSD estimation.

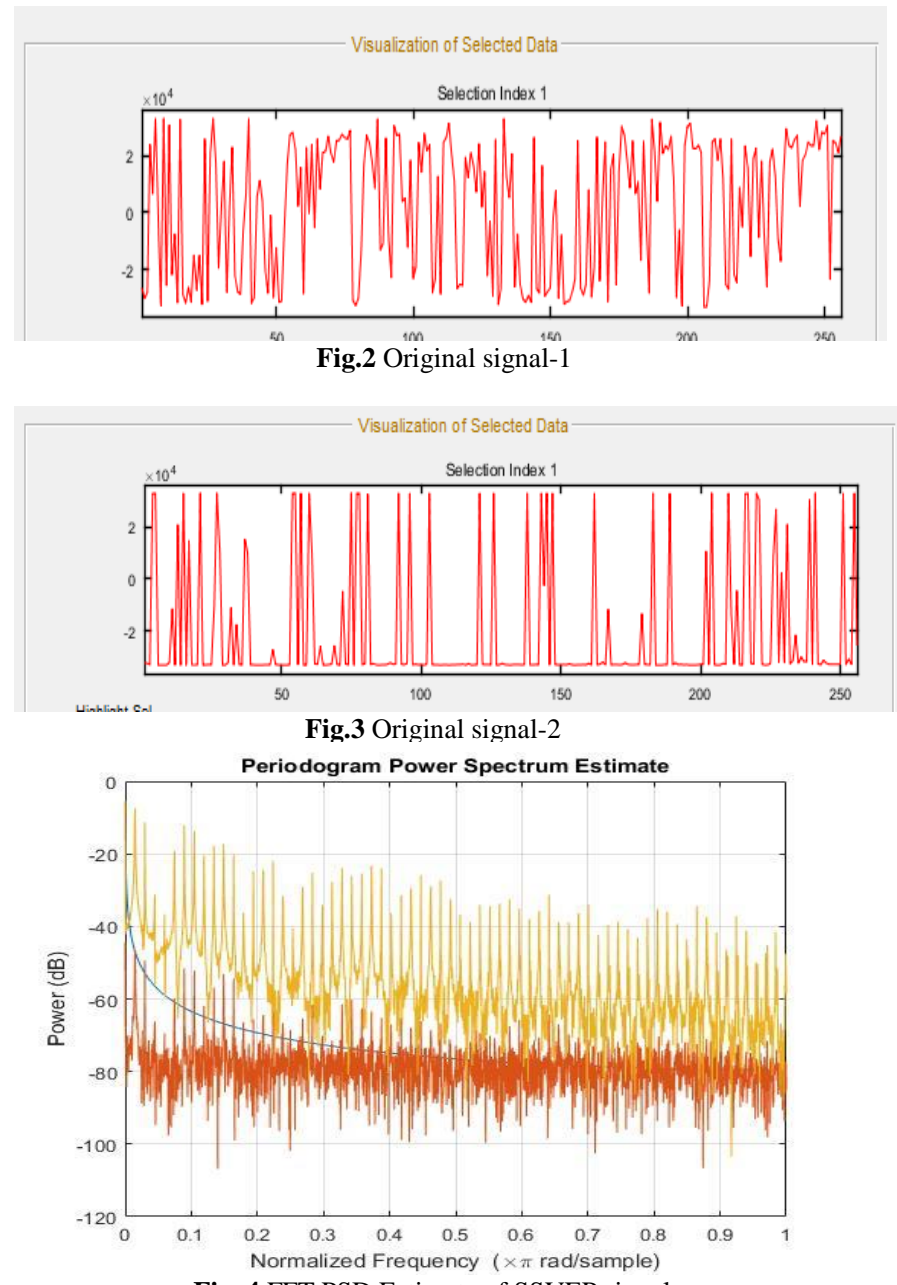

Fig. 4 FFT PSD Estimate of SSVEP signal

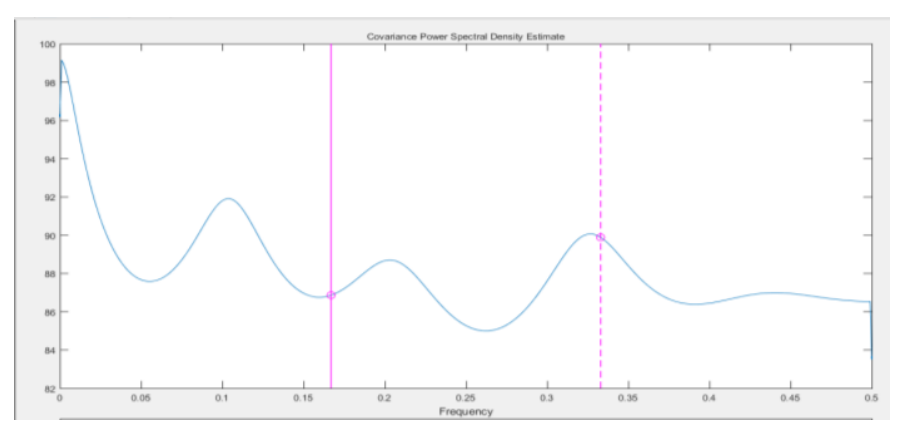

Fig. 5 Burg Covariance PSD Estimate of SSVEP signal 


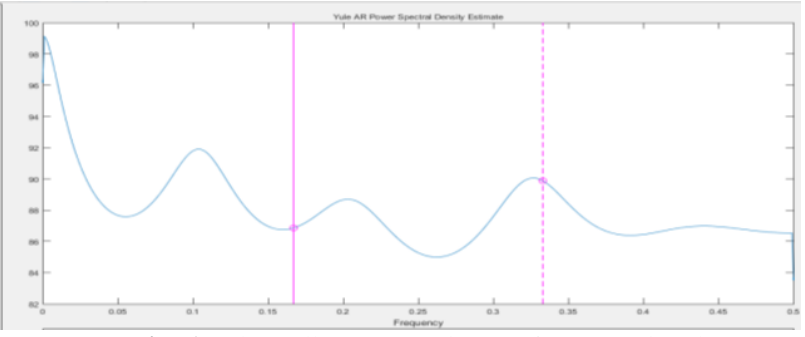

Fig. 6 Yule Walker PSD Estimate of SSVEP signal

The maximum frequency response lies in same frequency for both in Burg Covariance and Yule walker PSD estimation. Haar transform and Daubechies transforms are used for wavelet analysis for different subjects. Fig. 7 and Fig. 8 shows Haar and Daubechie transforms for sample signal 1. Fig. 9 and Fig. 10 for signal 2. From both sample signal analysis, it can be observed that Daubechies wavelets provides good results in SSVEP extraction.

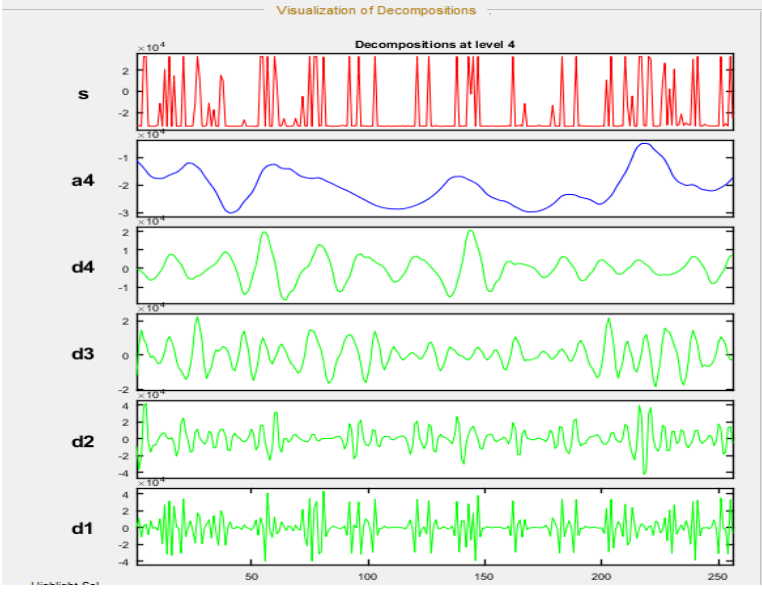

Fig. 10 Daubechies transformed Signal- 2

Table 1 shows decomposition levels (D1 to D4), residual signal (A4), mean value and energy for five subjects that obtained from Haar transform. Table 2 shows these values for Daubechies transform.

Table 1: Haar transform feature values for various subjects

\begin{tabular}{|c|c|c|c|c|c|}
\hline Subject & 1 & 2 & 3 & 4 & 5 \\
\hline Gender & $\mathrm{M}$ & $\mathrm{M}$ & $\mathrm{M}$ & $\mathrm{M}$ & $\mathrm{F}$ \\
\hline Mean & $-2.310 z^{-7 x}$ & $--2.063 \AA^{-7 x}$ & $-6.293 e^{+15 x}$ & $-1.462 \AA^{-7 x}$ & $-1.973 \AA^{-43}$ \\
\hline D1 & $; 33.56 \%$ & $\mathrm{H} 30.86 \%$ & I28.08\% & $224.61 \%$ & $224.24 \%$ \\
\hline D2 & 24.78 & $13.29 \%$ & $15.89 \%$ & $18.91 \%$ & $8.47 \%$ \\
\hline D3 & $16.91 \%$ & $5.75 \%$ & $8.79 \%$ & $9.71 \%$ & $5.56 \%$ \\
\hline D4 & $5.91 \%$ & $3.65 \%$ & $4.73 \%$ & $7.11 \%$ & $9.99 \%$ \\
\hline A4 & $18.84 \%$ & $40.46 \%$ & $31.16 \%$ & $40.16 \%$ & $51.74 \%$ \\
\hline Energy & $-1.353 \alpha^{-45}$ & $--2.505 \AA^{\mathrm{exz}}$ & $--1.863 e^{e x t}$ & $--1.526 \AA^{-2 \pi}$ & $-1.496 a^{\text {ext }}$ \\
\hline
\end{tabular}

Table 1: Daubechies transform feature values for various subjects

\begin{tabular}{|c|c|c|c|c|c|}
\hline Subject & 1 & 2 & 3 & 4 & 5 \\
\hline Gender & $\mathrm{M}$ & $\mathrm{M}$ & $\mathrm{M}$ & $\mathrm{M}$ & $\mathrm{F}$ \\
\hline Mean & $-2.3102^{-4 t}$ & $-2.063 a^{-7 z}$ & $-6.293 \AA^{7 x}$ & $-1.462 a^{-6 x}$ & $-1.973 \AA^{-4 \pi}$ \\
\hline D1 & $32.48 \%$ & $24.28 \%$ & $22.42 \%$ & $27.04 \%$ & $13.84 \%$ \\
\hline D2 & $15.26 \%$ & $14.96 \%$ & $12.43 \%$ & $12.88 \%$ & $10.68 \%$ \\
\hline D3 & $19.79 \%$ & $9.38 \%$ & $6.62 \%$ & $10.25 \%$ & $7.18 \%$ \\
\hline D4 & $6.16 \%$ & $4.94 \%$ & $5.53 \%$ & $8.63 \%$ & $1.35 \%$ \\
\hline A4 & $26.30 \%$ & $46.45 \%$ & $53.01 \%$ & $41.91 \%$ & $66.95 \%$ \\
\hline Energy & $-1.565 e^{-35}$ & $-2.505 \AA^{-3 x}$ & $-2.379 a^{-2 \pi x}$ & $-1.692 \pi^{-25 x}$ & $-2.125 \AA^{-13 x}$ \\
\hline
\end{tabular}

\section{Conclusion}

This proposed system focuses on various feature extraction procedures, applied on different subjects and processed data with MATLAB. Compared power spectral estimation and wavelet analysis, merits and demerits of each approach can be identified from the outcomes. Haar transform and Dabuchies transform are taken for wavelet analysis. FFT, Burg covariance and Yule walker analyzed for PSD estimation. It offers a theoretical reference of practical choice for BCI application

\section{References}

[1] Regan D, "Human brain electrophysiology: evoked potentials and evoked magnetic fields in science and medicine," J. Clin. Neurophysiol., 7: 450-451, 1990.

[2] Pasqualotto E, Federici S and Belardinelli M O, "Toward functioning and usable brain-computer interfaces (BCIs): a literature review,". Rehabil. Assist. Technol., 7: 89-103, 2012.

[3] Bin G Y, Gao X R, Yan Z, Hong B and Gao S K, "An online multichannel SSVEP-based brain-computer interface using a canonical correlation analysis method," J. Neural. Eng., 6: 046002, 2009. 
[4] Diez P F, Mut V A, Avila Perona E M and Laciar Leber E, "Asynchronous BCI control using high-frequency SSVEP," J. Neuroeng. Rehabil., 8: 39, 2011.

[5] Wong C M, Wang B Y, Wan F, Mak P U, Mak P I and Vai M I, "An improved phase-tagged stimuli generation method in steadystate visual evoked potential based brain-computer interface," Proc. 3rd Int. Conf. on Biomedical Engineering and Informatics, 2: 745 749, 2010.

[6] Muller-Putz G R and Pfurtscheller, "Control of an electrical prosthesis with an SSVEP-based BCI," IEEE Trans. Biomed. Eng., 55: 361-364, 2008.

[7] Li Zhao, Yong Sun, Research on Mobile Phone Dialing System Based on Steady-State Visual Evoked Potential, Chinese Journal of Biomedical Engineering, Vol.32,No.2,244-246,2013.

[8] Diez P F, Torres Müller S M, Mut V A, Laciar E, Avila E, BastosFilho T F and Sarcinelli-Filho M, "Commanding a robotic wheelchair with a high-frequency steady-state visual evoked potential based brain-computer interface," Med. Eng. Phys., 35: 1155-1164, 2013.

[9] Yan B, Li Z, Li H, Yang G and Shen H, "Research on brain- computer interface technology based on steady state visual evoked potentials," Proc. 4th Int. Conf. on Bioinformatics and Biomedical Engineering, 1-4, 2010.

[10] Bian Y, Li H W, Zhao L, Yang G H and Geng L Q, "Research on steady state visual evoked potentials based on wavelet packet technology for brain-computer interface," Proc. Eng., 15: 2629-2633, 2011.

[11] Zhang Z, Li X and Deng Z, "A CWT-based SSVEP classification method for brain-computer interface system," Proc. Int. Conf. on Intelligent Control and Information Processing, 43-48, 2010.

[12] P L Lee, Chang H C, Hsieh T Y, Deng H T and Sun C W, "A brainwave-actuated small robot car using ensemble empirical mode decomposition-based approach," IEEE Trans. Syst. Man Cybern. Syst. Hum., 42: 1053-1064, 2012.

[13] Garcia-Molina G, Zhu D H and Abtahi S, "Phase detection in a visual-evoked-potential based brain computer interface," Proc. 18th European Signal Processing Conf., 949-953, 2010.

[14] Zhu D H, Garcia-Molina G, Mihajlović V and Aartsl R M, "Phase synchrony analysis for SSVEP-based BCIs," Proc. 2nd Int. Conf. on Computer Engineering and Technology, 2: V2329- V2333, 2010.

[15] Zhao L, Yuan P X, Xiao L T, Meng Q G, Hu D F and Shen H, "Research on SSVEP feature extraction based on HHT," Proc. 7th Int. Conf. on Fuzzy Systems and Knowledge Discovery, 5: 2220-2223, 2010

[16] Zhu D, Garcia-Molina G, Mihajlović V and Aarts R M, "Online BCI implementation of high-frequency phase modulated visual stimuli," Universal Access in Human- Computer Interaction. Users Diversity, 6766: 645-654, 2011.

[17] Friman O, Luth T, Volosyak I and Graser A, "Spelling with steadystate visual evoked potentials," Proc. 3rd Int. IEEE/EMBS Conf. on Neural Eng., 354-357, 2007.

[18] Müller S M T, A. M. F. L. M. De Sá, Bastos-Filho T F and Sarcinelli-Filho M, "Spectral techniques for incremental SSVEP analysis applied to a BCI implementation," Proc. 5th Latin American Congress on Biomedical Engineering, 33: 1090-1093, 2013

[19] Muller S M T, Bastos T F and Sarcinelli M, "Incremental SSVEP analysis for BCI implementation," Proc. Annual Int. Conf. of the IEEE Engineering in Medicine and Biology Society, 3333-3336, 2010.

[20] Chang H C, Deng H T, Lee P L, Wu C H and Shyu K K, "Realtime control of an SSVEP-actuated remote-controlled car," Proc. SICE Annual Conf., 1884-1887, 2010.

[21] https://physionet.org/physiobank/database/mssvepdb 